\title{
STXBP1-associated neurodevelopmental disorder: a comparative study of behavioural characteristics
}

\author{
Sinéad O'Brien ${ }^{1}$, Elise Ng-Cordell ${ }^{1,2}$, The DDD Study ${ }^{3}$, Duncan E. Astle ${ }^{1}$, Gaia Scerif ${ }^{2}$ and Kate Baker ${ }^{1,4^{*}}$ (D)
}

\begin{abstract}
Background: De novo loss of function mutations in STXBP1 are a relatively common cause of epilepsy and intellectual disability (ID). However, little is known about the types and severities of behavioural features associated with this genetic diagnosis.

Methods: To address this, we collected systematic phenotyping data encompassing neurological, developmental, and behavioural characteristics. Participants were 14 individuals with STXBP1-associated neurodevelopmental disorder, ascertained from clinical genetics and neurology services UK-wide. Data was collected via standardised questionnaires administered to parents at home, supplemented by researcher observations. To isolate discriminating phenotypes, the STXBP1 group was compared to 33 individuals with pathogenic mutations in other ID-associated genes (ID group). To account for the potential impact of global cognitive impairment, a secondary comparison was made to an abilitymatched subset of the ID group (low-ability ID group).
\end{abstract}

Results: The STXBP1 group demonstrated impairments across all assessed domains. In comparison to the ID group, the STXBP1 group had more severe global adaptive impairments, fine motor difficulties, and hyperactivity. In comparison to the low-ability ID group, severity of receptive language and social impairments discriminated the STXBP1 group. A striking feature of the STXBP1 group, with reference to both comparison groups, was preservation of social motivation.

Conclusions: De novo mutations in STXBP1 are associated with complex and variable neurodevelopmental impairments. Consistent features, which discriminate this disorder from other monogenic causes of ID, are severe language impairment and difficulties managing social interactions, despite strong social motivation. Future work could explore the physiological mechanisms linking motor, speech, and social development in this disorder. Understanding the developmental emergence of behavioural characteristics can help to focus clinical assessment and management after genetic diagnosis, with the longterm aim of improving outcomes for patients and families.

Keywords: STXBP1, Epilepsy, Intellectual disability, Language, Social

\section{Background}

It is increasingly possible to identify the genetic cause of severe neurodevelopmental disorders, with potential diagnostic yields now exceeding 50\% [1]. For each rare genetic cause, there is an ongoing imperative to characterise associated medical and behavioural features in order to provide

\footnotetext{
* Correspondence: Kate.Baker@mrc-cbu.cam.ac.uk

${ }^{1}$ MRC Cognition and Brain Sciences Unit, University of Cambridge, 15

Chaucer Road, Cambridge CB2 7EF, UK

${ }^{4}$ Department of Medical Genetics, University of Cambridge, Cambridge

Biomedical Campus, Hills Road, Cambridge CB2 OXY, UK

Full list of author information is available at the end of the article
}

evidence-based prognostic advice, targeted family support, and ultimately personalised interventions.

Syntaxin-binding protein 1 (STXBP1, formally known as MUNC18-1) is part of the SEC1 family of membranetrafficking proteins that interact with SNARE proteins to facilitate the docking of synaptic vesicles at presynaptic active zones to enable neurotransmission [2]. STXBP1 is expressed ubiquitously throughout the brain and neocortex during development and postnatal life. De novo STXBP1 variants were initially identified in five patients as a cause of Ohtahara syndrome, also known as early infantile epileptic encephalopathy [3]. Thereafter, the epilepsy

(c) The Author(s). 2019 Open Access This article is distributed under the terms of the Creative Commons Attribution 4.0 International License (http://creativecommons.org/licenses/by/4.0/), which permits unrestricted use, distribution, and 
spectrum associated with STXBP1 variants expanded to include infantile spasms, West syndrome, Dravet syndrome, Lennox-Gastaut syndrome, and various other types of childhood-onset epilepsy [4-8]. The estimated prevalence of de novo $S T X B P 1$ variants in severe childhood epilepsy is $3 \%$ [9]. In parallel, STXBP1 variants have been discovered in individuals broadly ascertained for neurodevelopmental disorders, with and without epilepsy [7, 10-14]. The prevalence of STXBP1 variants amongst individuals with unspecified developmental disorders is $0.25-0.5 \%[15,16]$. Given the $1 \%$ prevalence of intellectual disability (ID), the numbers of individuals diagnosed with $S T X B P 1$-associated neurodevelopmental disorder will rise substantially over the coming years.

Collation of clinical information has highlighted an association between STXBP1 variants and complex long-term neurodevelopmental impairments, in patients with and without epilepsy. Existing case series suggest that there is considerable variability in the types and severities of neurodevelopmental problems associated with $S T X B P 1$ variants. Reported problems include movement impairments such as ataxia and tremor, language delay and behavioural symptoms including hyperactivity, stereotypies, and autistic features [7, 9, 13, 17-20]. However, despite the increasing number of patients diagnosed with STXBP1 variants, no study has carried out post-diagnostic, standardised assessments to systematically characterise developmental and behavioural aspects of the STXBP1-associated phenotype. These data are necessary to provide families with valid information about the types and severities of neurodevelopmental difficulties that can be expected for an individual diagnosed with an STXBP1 variant. Hence, the primary aim of the current study was to describe in detail the neurodevelopmental phenotype of children and adolescents with STXBP1 variants, ascertained as broadly as possible.

The secondary aim of the study was to relate neurodevelopmental characteristics associated with STXBP1 variants to existing basic science literature, including animal models. STXBP1 plays a role in calcium-dependent, short-term synaptic facilitation, which may be particularly relevant to learning [21]. STXBP1 loss of function mutations lead to broad impairments in synaptic physiology that nonetheless result in specific learning deficits and behavioural features. In cultured neurons from human embryonic stem cells, homozygous mutations impede synaptic transmission which results in neurodegeneration, whereas heterozygous mutations have a milder impact [21]. Homozygous knockout mice have shown that, despite normal brain assembly, lack of STXBP1 expression inhibits pre-synaptic events which leads to synaptic degeneration, neuronal apoptosis, and early fatality [22-24]. In contrast, heterozygous STXBP1 mouse models have reduced protein expression and stability, but structurally normal synaptogenesis. Mice demonstrate a seizure phenotype characterised by twitches, jerks, and jumps which often coincided with EEG spikewave discharges [25]. Currently, there is no systematic, comparative data available on the human behavioural phenotype to verify whether behavioural results in STXBP1 mouse models [26-28] recapitulate the human disorder. To achieve this, we have compared individuals with STXBP1 variants to participants with equivalently severe ID, to determine which if any characteristics are consistently observed and are specific to this group. This comparison can add specificity to the prognostic information available to families and clinicians and set hypotheses for future investigation of molecular, neural, and cognitive mechanisms.

\section{Methods}

\section{Participants and recruitment}

Individuals who had previously been diagnosed with a pathogenic de novo variant in $S T X B P 1$ were identified via clinical genetics services, paediatric neurology services, and clinical testing laboratories. Responsible clinicians were provided with a recruitment pack for each potential participant, and 14 families volunteered to participate in the study. Eight participants had been diagnosed with an STXBP1 variant via the Deciphering Developmental Disorders research study [16], and the remainder had been diagnosed via a clinical testing pathway. For both testing pathways, pathogenicity of variants had been evaluated by the regional genetics service according to ACMG guidelines and local confirmation procedures. The same recruitment procedure was followed for the comparison sample of individuals with neurodevelopmental disorder associated with single nucleotide variants in other ID-associated genes. Participants were recruited with the assistance of 10 regional genetics centres.

\section{Phenotyping assessments}

All 14 participants were assessed in their homes. A standardised, study-specific, structured medical history interview was conducted, followed by the Vineland Adaptive Behaviour Scales, survey interview form [26]. Parents or carers were also given age-appropriate questionnaires to complete which included the Developmental Behavioural Checklist (DBC) [27], Conners-3 short-form [28], Social Responsiveness Scale (SRS-2) [29], and the Movement ABC Parent Checklist [30]. The Movement Assessment Battery for Children (MABC-2) was used to directly assess participants' manual dexterity, aiming and catching, and balance [30]. Detailed notes and video recordings were obtained in order to document qualitative observations of each participant.

\section{Data completion rates}

For descriptive reporting, data from all STXBP1 participants are included $(n=14)$ (Additional file 1). For comparative analyses, analysis was restricted to a consistent STXBP1 group for whom a complete questionnaire 
dataset was available ('STXBP1 full dataset group', $n=8$ ). Partial data collection was achieved for younger participants (protocol only appropriate for ages 4 and above) and for three participants without a fully completed parent questionnaire pack.

\section{Analysis}

Raw scores from questionnaire data were standardised to published normative data. Non-parametric comparisons were conducted throughout (Mann-Whitney $U$ statistics).

\section{Results}

\section{Demographics of STXBP1 and comparison groups} (Table 1)

The STXBP1 group comprised 14 individuals with a confirmed pathogenic, de novo variant in STXBP1. The ID comparison group comprised 33 individuals with confirmed pathogenic or likely pathogenic de novo or inherited variants in other ID-associated genes (Additional file 2). The STXBP1 full dataset and ID groups did not differ in age, gender or socioeconomic status (SES) distributions. On average, the STXBP1 group had more severe global adaptive impairments than the ID comparison group (Mann-Whitney $U=43.5, p=0.004$ ), with more severe deficits in all adaptive behaviour subscales except for gross motor function (Table 2). To identify specific behavioural characteristics not explained by these global impairments, secondary analyses were carried out between the STXBP1 full dataset group and a low-ability subset of the ID comparison group, selected to match the range of Vineland Adaptive Behaviour Composite scores. The STXBP1 full dataset and low-ability ID comparison groups did not differ in age, gender, or SES representation.
STXBP1 group-medical, neurological, and neurodevelopmental characteristics General health

Duration of pregnancy ranged from 32 to 42 weeks. No abnormal ultrasounds or threatened miscarriages were reported. However, five mothers reported persistent vomiting throughout pregnancy. There were concerns about foetal well-being during the birth of six of the participants. Four participants were born by Caesarean section. Only three cases subsequently spent time in SCBU (with none requiring neonatal intensive care). Three individuals were readmitted to hospital within the first month of life for investigation of suspected seizures.

A large proportion of parents (85\%) reported feeding issues during infancy and early childhood, common problems being reflux, choking, and difficulty moving solids. Sleep difficulties were reported in four cases during infancy, characterised by problems falling and staying asleep. In two cases, sleep difficulties persisted into adolescence.

Parents also reported a range of sensory issues with regard to hearing, sight, and touch. Six parents reported sight issues including; ptosis, misshapen eye, difficulty focusing, excessive blinking, and long-sightedness. Three parents reported hearing issues: one participant suffered from recurrent ear infections, one had glue ear in both ears, and one participant required hearing aids. A diverse range of sensory issues involving touch and sound were reported in eight cases (3: touch, 3: sound, 2: touch and sound). Three participants enjoyed sensory feedback from sensory objects and people (e.g., hugging and rough play), whereas two did not. One child appeared to enjoy high-pitched sounds such as babies crying, whereas four parents noticed that their children were averse to certain loud sounds such as hoovers, fireworks, and weather. One of these children had received a diagnosis of hyperacusis.

Table 1 Demographic information

\begin{tabular}{llllll}
\hline Demographic measure & & Group & & \\
\cline { 3 - 5 } & & STXBP1 all $(n=14)$ & STXBP1 full dataset $(n=8)$ & ID all $(n=33)$ & ID low ability $(n=8)$ \\
\hline Age & Mean (SD) & $9.93(5.837)$ & $13.63(2.326)$ & $13.91(5.553)$ & $14.88(3.271)$ \\
& Range (years) & $1-17$ & $10-17$ & $5-25$ & $8-18$ \\
Gender & \% females( $n)$ & $71.4(10)$ & $62.5(5)$ & $51.5(17)$ & $62.5(5)$ \\
SES** & Median (SD, range) & $6.5(2.51,2-10)$ & $7(1.96,4-10)$ & $6(2.33,1-10)$ & $6.5(1.98,3-9)$ \\
Epilepsy (ever) & $\%(n)$ & $92.9(13)$ & $87.5(7)$ & $39.4(13)$ & $50(4)$ \\
Epilepsy (current) & $\%(n)$ & $92.3(12)$ & $85(6)$ & $62(8)$ & $75(3)$ \\
Epilepsy age of onset & Mean (SD) & $49.67(67.059)$ & $84.14(70.115)$ & $70.73(76.352)$ & $12.67(9.866)$ \\
& Range (months) & $1-156$ & $1-156$ & $1-204$ & $6-24$ \\
Global intellectual ability & Mean (SD) & $45.86(14.992)$ & $41.0(12.13)$ & $58.3(15.661)^{\#}$ & $48.0(9.592)$ \\
& Range & $26-69$ & $31-67$ & $20-96$ & $36-68$
\end{tabular}

**SES calculated using 'English Indices of Deprivation' (2015)

\#Comparison to STXBP1 full dataset group, Mann-Whitney $U$ test, $P<0.05$ 
Table 2 Adaptive function

\begin{tabular}{|c|c|c|c|c|c|}
\hline \multirow{2}{*}{\multicolumn{2}{|c|}{$\begin{array}{l}\text { Vineland (standard score) } \\
\text { Domains } \\
\text { Subdomains }\end{array}$}} & \multicolumn{4}{|l|}{ Groups } \\
\hline & & \multirow{2}{*}{$\begin{array}{l}\text { STXBP1 all }(n=14) \\
42.64(11.5)\end{array}$} & \multirow{2}{*}{$\begin{array}{l}\text { STXBP1 full dataset }(n=8) \\
43.38(12.1)\end{array}$} & \multirow{2}{*}{$\begin{array}{l}\text { ID all }(n=33) \\
59.15(18.6)^{\#}\end{array}$} & \multirow{2}{*}{$\begin{array}{l}\text { ID low ability }(n=8) \\
47.50(7.6)\end{array}$} \\
\hline Communication & Mean (SD) & & & & \\
\hline & Range & $30-67$ & $33-67$ & $21-100$ & $38-61$ \\
\hline \multirow[t]{2}{*}{ Receptive } & Mean (SD) & $4.82(2.7)$ & $4.75(2.6)$ & $8.70(3.1)^{\#}$ & $7.13(1.5)^{\#}$ \\
\hline & Range & $2-10$ & $2-10$ & $1-16$ & $5-10$ \\
\hline \multirow[t]{2}{*}{ Expressive } & Mean (SD) & $3.55(2.7)$ & $3.38(2.8)$ & $6.88(4.0)^{\#}$ & 3.00 (1.9) \\
\hline & Range & $1-8$ & $1-8$ & $1-17$ & $1-6$ \\
\hline \multirow[t]{2}{*}{ Written } & Mean (SD) & $5.55(1.8)$ & $5.38(1.8)$ & $7.97(3.4)^{\#}$ & $5.88(1.0)$ \\
\hline & Range & $4-9$ & $4-9$ & $3-20$ & $5-8$ \\
\hline \multirow[t]{2}{*}{ Daily living skills } & Mean (SD) & 45.57 (19.7) & 38.38 (15.5) & $57.36(18.2)^{\#}$ & 43.00 (13.3) \\
\hline & Range & $25-77$ & $25-73$ & $21-98$ & $30-71$ \\
\hline \multirow[t]{2}{*}{ Personal } & Mean (SD) & $4.71(4.5)$ & $2.88(3.8)$ & $6.18(4.3)^{\#}$ & $2.75(2.6)$ \\
\hline & Range & $1-12$ & $1-12$ & $1-19$ & $1-8$ \\
\hline \multirow[t]{2}{*}{ Domestic } & Mean (SD) & $6.43(4.3)$ & 4.38 (3.3) & $8.73(3.8)^{\#}$ & $5.63(3.3)$ \\
\hline & Range & $2-13$ & $2-12$ & $3-17$ & $3-13$ \\
\hline \multirow[t]{2}{*}{ Community } & Mean (SD) & 4.86 (3.9) & $3.00(1.9)$ & $6.18(3.6)^{\#}$ & 3.88 (2.6) \\
\hline & Range & $1-12$ & $1-7$ & $1-13$ & $1-9$ \\
\hline \multirow[t]{2}{*}{ Socialisation } & Mean (SD) & $52.29(12.1)$ & $47.75(8.4)$ & $63.30(16.5)^{\#}$ & $56.13(9.2){ }^{\#}$ \\
\hline & Range & $34-75$ & $40-67$ & 20-108 & $46-75$ \\
\hline \multirow[t]{2}{*}{ Interpersonal } & Mean (SD) & 6.00 (3.3) & $4.63(2.1)$ & $7.94(3.0)^{\#}$ & $6.63(1.8)$ \\
\hline & Range & $2-13$ & $2-8$ & $1-15$ & $4-10$ \\
\hline \multirow[t]{2}{*}{ Play and leisure } & Mean (SD) & $4.93(3.4)$ & $3.63(3.2)$ & $7.76(3.6)^{\#}$ & $5.00(3.7)$ \\
\hline & Range & $1-11$ & $1-11$ & $1-15$ & $1-11$ \\
\hline \multirow[t]{2}{*}{ Coping skills } & Mean (SD) & $6.79(1.8)$ & $6.00(1.3)$ & $9.30(3.1)^{\#}$ & $8.00(1.3)^{\#}$ \\
\hline & Range & $4-9$ & $5-8$ & $6-20$ & $6-10$ \\
\hline \multirow[t]{2}{*}{ Motor } & Mean (SD) & $60.43(16.4)$ & 63.25 (17.4) & $69.03(12.4)$ & $62.63(5.6)$ \\
\hline & Range & $37-97$ & 40-97 & 56-107 & $56-72$ \\
\hline \multirow[t]{2}{*}{ Gross } & Mean (SD) & 8.50 (2.8) & 9.63 (3.0) & 9.58 (1.8) & 9.00 (1.5) \\
\hline & Range & $5-15$ & $6-15$ & $7-16$ & $7-11$ \\
\hline \multirow[t]{2}{*}{ Fine } & Mean (SD) & $8.29(4.1)$ & 8.00 (3.1) & $10.09(3.0)^{\#}$ & $8.50(0.9)$ \\
\hline & Range & $4-17$ & $4-14$ & $7-20$ & $7-10$ \\
\hline
\end{tabular}

"Comparison to STXBP1 full dataset group, Mann-Whitney $U$ test, $P<0.05$

\section{Neurological histories}

Thirteen STXBP1 participants have a history of seizures, diagnosed as epilepsy by a paediatric neurologist. The age of seizure onset varied from just after birth to 13 years of age. Half of the STXBP1 participants $(n=7)$ had a seizure in their first year of life. Four of these participants had a seizure within the first 2 months of life. For the remaining six individuals with epilepsy, the age of onset of seizures was broadly spread across childhood. Three participants experienced their first seizure during the peripubertal phase. The type of seizure also varied. One participant was diagnosed with infant encephalopathy, two had tonic- clonic seizures, and six experienced focal seizures. Seizure phenotypes were mixed or uncertain for the remaining participants. Epilepsy has been treated with a wide range of anti-epileptic drugs (AEDs), the most frequently used was levetiracetam $(n=4)$. Whilst seizure frequency was generally reduced on treatment, no participant was reported to be completely seizure free during the months prior to research assessment. One participant experienced infrequent seizures (approximately three between 5 and 14 years of age) but was not on medication. Four participants also experienced tremors in their hands, which were observed by the researcher during assessments. 


\section{Developmental milestones}

Thirteen participants learned to sit independently from 7 to 42 months. Seven out of thirteen sat independently before 10 months, which is within the normal to mildly delayed range, whilst the remainder were clearly delayed. The youngest child in the sample (1-year-old) had extremely low muscle tone and was not able to sit independently at the time of assessment. Four children did not learn to crawl but 'bum-shuffled' or 'bunny hopped'. Nine participants were walking independently at the time of assessment. However, eight acquired this skill late: between 25 and 60 months old. One participant, aged 14, could walk a few steps but required constant support. Three participants between 1.9 and 6.8 years of age had not yet learned to walk.

Speech and language acquisition were delayed in all cases. Eight participants currently use some verbal communication, with age of first words being between 18 and 156 months (13 years). The age of participants not currently using verbal communication is between 1.1 and 15 years. Of the four participants who can form simple phrases or sentences, three of these acquired this skill late, after 5.5 years of age.

\section{Current motor abilities}

Vineland gross and fine motor subscale scores reflect the extent of impairment and range of motor abilities within the sample. Gross motor standardised scores ranged between 6 and 15: from severely impaired to average for age (maximum score 24, normative population mean $=15, \mathrm{SD}=3$ ). The participant with the highest score could run, jump, hop, skip, and kick, throw, and catch a ball, whereas, at age 6 , the participant with the score was unable to pull themselves to a standing position. Similarly, fine motor standard scores ranged from 4 to 17. Participants with greater fine motor abilities could draw shapes freehand and build three-dimensional structures whereas three participants were unable to turn pages or stack blocks.

The MABC-2 Parent Checklist provided a parent rating of their child's motor abilities in predictable and unpredictable environments. In addition, a 13-item subscale asks parents to indicate any non-motor factors that may affect their child's movement. Over $85 \%$ of parents in the STXBP1 group reported that being overactive, distractible, impulsive, disorganised, and hesitant contributed to their child's motor skills difficulties. In addition, $62.5 \%$ of children became anxious in stressful, movement-related situations. All participants attempted MABC-2 motor tasks but only one participant was able to complete the full protocol enabling standardised scoring.

\section{Current adaptive and communication abilities}

The global adaptive function was estimated via the Vineland Adaptive Behaviour Scales (parent interview) composite score. Seven participants had scores in the severe ID range and seven participants were in the mild to moderate ID range. Notably, three participants under 2 years of age scored in the mild ID range, which may indicate increasing diversion from developmental expectations with age.

Vineland Communication standard scores have a mean of 100 and a standard deviation of 15 . Participants scored between 33 and 67 (i.e. moderately to severely impaired range). The $S T X B P 1$ participants' receptive abilities ranged from an inability to listen and attend to speech to being able to recall and carry out instructions given 5 min previously. Expressive ability ranged from difficulty with prespeech expression to engaging in interactive speech.

\section{Current emotional and behavioural difficulties}

The Developmental Behavioural Checklist (DBC) was completed by parents to characterise the current behavioural and emotional difficulties they observe in their children (participants over the age of 5). Seven out of eight STXBP1 participants had a DBC Total Problem Behaviour Score above the clinical cut-off ( $T$ score 46$)$ indicative of the likely presence of psychopathology. Behaviours such as biting, spitting, ripping things, hitting, and running away from caregivers were common, and parents reported demanding situations and frustration. Two parents remarked that these behaviours had become increasingly difficult to manage following the onset of puberty. High levels of anxiety associated with specific causes such as fireworks, adverse weather, hoovers, lawnmowers, and the emotions of others were reported in five cases (4 females, 1 male). Six parents also reported that their child rarely cried and did not appear to feel pain.

Parents completed the Conners Short Form to assess the characteristics of ADHD and comorbid problems in the sample. The STXBP1 group showed very elevated scores on the inattention, hyperactivity, learning problems, and peer relations subscales. Two participants had a $T$ score in the normal range for executive functions and five participants scored within the normal range on the aggression subscale.

\section{Current social functioning}

Vineland socialisation subscale scores indicated that the STXBP1 group demonstrate social adaptive impairments. The Social Responsiveness Scale (SRS-2) was used to characterise atypical social processes linked to autism spectrum features in the sample. Three participants had an SRS total score within the mild to moderately impaired range and five scored above 76 , which is indicative of severe social impairment likely to meet criteria for an autism spectrum diagnosis. Participants' scores fell within the moderate to severe range on social awareness, social cognition, social 
communication and restricted interests and repetitive behaviours subscales. In contrast, scores on the social motivation subscale were notably preserved and within the normal range. Parents typically described a social profile characterised by self-confidence in social settings and an interest in engaging with peers and adults. The study researchers also observed this strong interest in social interaction, despite limited communication and social skills as assessed by Vineland scales.

\section{Comparison of behavioural features in the STXBP1 full dataset and ID groups Motor function}

There was no difference in gross motor skills between groups as assessed via Vineland Adaptive Behaviour Scales (Table 2) and Movement ABC (Table 3). However, the $S T B X P 1$ group had poorer Vineland fine motor subscale scores when compared to the ID group $(U=74.0$, $p=0.053)$. There was no difference between groups in how parents evaluated the contextual factors influencing children's motor performance.

\section{Emotional and behavioural difficulties}

The STXBP1 and ID groups did not differ on DBC total problem behaviour scores or subscale scores (stratified for ID severity), indicating that emotional and behavioural difficulties in general are not more prevalent or severe in the STXBP1 group than expected for intellectual disability due to other causes (Table 3). Anxiety and disruptive behaviours were prominent problems for some but not all individuals within the STXBP1 group; however, group mean DBC anxiety subscale scores did not differ from the ID comparison group. On the Conners scales, the STXBP1 group were rated as significantly more hyperactive/impulsive compared to the ID group $(U=52.0, p=0.026)$. In summary, the $S T X B P 1$ group demonstrated similar total problem behaviour scores to the ID comparison group, with variation within the group in the severity and types of problems reported for each individual. High hyperactivity/impulsivity scores were more consistent within the STXBP1 group and on average were more severe than reported for individuals with other monogenic causes of ID.

\section{Social functioning}

There was a significant difference between STXBP1 and the ID groups on Vineland Socialisation scores: the ID group on average display stronger skills on all three subscales-interpersonal relations, play and leisure, and coping skills. The groups did not differ on SRS total score or social awareness, social cognition, social communication and restricted interests and repetitive behaviours (RRB) subscales. However, the ID comparison group were more impaired on social motivation, compared to the
STXBP1 group who fell within the 'normal' range for this subscale $(U=74.0, p=0.06)$. In summary, the STXBP1 group demonstrated an atypical profile of social functioning in comparison to ID in general, with more severely impaired everyday social behaviour (on Vineland scales), equivalently impaired social cognition (on SRS), but preserved social motivation.

\section{Comparison of behavioural features in the STXBP1 full dataset and low-ability ID groups}

To explore the specificity of behavioural characteristics associated with STXBP1 mutation in more detail, secondary comparisons were made to a subset of the ID comparison group selected to match the range of global adaptive abilities within the STXBP1 group. Although the two groups had comparable global adaptive function scores, communication impairments were more severe in the STXBP1 group. The comparison group on average demonstrated stronger receptive than expressive abilities whereas the STXBP1 group showed severe restriction of both receptive and expressive abilities (with significant difference between groups in receptive score: $U=11.0$, $p=0.025)$. Groups did not differ in gross or fine motor abilities. Behavioural problems assessed via the DBC (total, subscales) did not differ between groups. The STXBP1 group also did not differ from the low-ability ID group in Conners hyperactivity or impulsivity scores, indicating that although these difficulties are a consistent problem area for the STXBP1 group, they are not more impaired in these domains than other individuals with equivalent global impairments. Comparison of Vineland scores indicated that everyday social behaviours were more impaired in the STXBP1 group in comparison to the low-ability ID group (socialisation domain: $U=11.0$, $p=0.027$, coping: $U=9.0, p=0.014$ ). The analysis of SRS scores (see Fig. 1) indicated a stable pattern of results whether comparing to the whole sample ID group or low-ability ID group-total SRS scores did not differ, social cognitive scores did not differ, restricted and repetitive behaviour scores did not differ, but social motivation was significantly preserved in the STXBP1 group $(U=9.5, p=0.018)$.

\section{Discussion}

In this study, we systematically assessed the behavioural characteristics of children and adolescents with variants in STXBP1, aiming to improve prognostication and management for individuals with this rare genetic diagnosis. Participants in the STXBP1 group demonstrated complex and persistent difficulties across multiple domains of everyday function and emotional-behavioural development. Consistent problems within the group included severe communication impairments and hyperactivity-impulsivity. Some participants were greatly troubled by anxiety 
Table 3 Emotional and behavioural characteristics

\begin{tabular}{|c|c|c|c|c|}
\hline \multirow[t]{2}{*}{ Outcome measure } & & \multicolumn{3}{|l|}{ Groups } \\
\hline & & STXBP1 full dataset $(n=8)$ & ID all $(n=33)$ & ID low ability $(n=8)$ \\
\hline \multicolumn{5}{|l|}{ MABC parent checklist } \\
\hline \multirow[t]{2}{*}{ Motor competence (static environments) } & Mean (SD) & $24.5(12.3)$ & $18.45(9.2)$ & $23.00(5.6)$ \\
\hline & Range & $3-42$ & $1-37$ & $11-31$ \\
\hline \multirow[t]{2}{*}{ Motor competence (dynamic environments) } & Mean (SD) & $26.00(11.9)$ & $22.12(8.8)$ & $25.5(6.8)$ \\
\hline & Range & $3-43$ & $3-39$ & $14-34$ \\
\hline \multirow[t]{2}{*}{ Total motor score } & Mean (SD) & $50.5(23.6)$ & $40.61(17.2)$ & $48.5(10.9)$ \\
\hline & Range & $6-85$ & $6-74$ & $35-62$ \\
\hline \multicolumn{5}{|l|}{ Conners ( $T$ score) } \\
\hline \multirow[t]{2}{*}{ Inattention } & Mean (SD) & $84.75(7.5)$ & $79.19(12.0)$ & $86.00(5.3)$ \\
\hline & Range & $72-92$ & $55-90$ & $79-90$ \\
\hline \multirow[t]{2}{*}{ Hyperactivity } & Mean (SD) & $85.25(6.5)$ & $72.37(15.0)^{\#}$ & $77.25(13.2)$ \\
\hline & Range & $73-90$ & $40-90$ & $56-90$ \\
\hline \multirow[t]{2}{*}{ Learning problems } & Mean (SD) & $86.38(8.3)$ & $79.44(10.9)$ & $86.13(5.0)$ \\
\hline & Range & $66-90$ & $57-90$ & $78-90$ \\
\hline \multirow[t]{2}{*}{ Executive functions } & Mean (SD) & $76(14.8)$ & $64.56(14.4)$ & $66.25(13.3)$ \\
\hline & Range & $54-90$ & $43-90$ & $48-83$ \\
\hline \multirow[t]{2}{*}{ Aggression } & Mean (SD) & $59.88(17.4)$ & $52.3(10.5)$ & $47.5(4.8)$ \\
\hline & Range & $44-90$ & $44-83$ & $44-58$ \\
\hline \multirow[t]{2}{*}{ Peer relations } & Mean (SD) & $84.13(7.2)$ & $80.33(15.0)$ & $81.38(16.1)$ \\
\hline & Range & $72-90$ & $45-90$ & $45-90$ \\
\hline \multicolumn{5}{|l|}{ DBC (percentile/stratified by ID severity) } \\
\hline \multirow[t]{2}{*}{ Disruptive } & Mean (SD) & $68.25(24.3)$ & $50.91(28.8)$ & $54.00(25.2)$ \\
\hline & Range & $28-90$ & $4-98$ & $26-90$ \\
\hline \multirow[t]{2}{*}{ Self-absorbed } & Mean (SD) & $73.75(19.2)$ & $70.12(25.8)$ & $77.75(17.9)$ \\
\hline & Range & $34-94$ & $10-100$ & $40-100$ \\
\hline \multirow[t]{2}{*}{ Communication } & Mean (SD) & $75.5(17.8)$ & $76.42(23.1)$ & $78.00(18.8)$ \\
\hline & Range & $48-96$ & $6-100$ & $40-92$ \\
\hline \multirow[t]{2}{*}{ Anxiety } & Mean (SD) & $42.5(24.5)$ & $58.3(26.9)$ & $66.25(30.1)$ \\
\hline & Range & $12-76$ & $10-100$ & $10-96$ \\
\hline \multirow[t]{2}{*}{ Social relations } & Mean (SD) & $49.5(22.8)$ & $58.85(28.0)$ & $57.00(27.3)$ \\
\hline & Range & $24-96$ & $12-100$ & 24-98 \\
\hline \multirow[t]{2}{*}{ Total problems } & Mean (SD) & $73.50(24.1)$ & $63.94(27.4)$ & $67.25(22.3)$ \\
\hline & Range & 30-94 & $0-100$ & $38-98$ \\
\hline \multicolumn{5}{|l|}{ SRS ( $T$ score) } \\
\hline \multirow[t]{2}{*}{ Social awareness } & Mean (SD) & $78.75(5.9)$ & $73.64(11.2)$ & $76.38(12.3)$ \\
\hline & Range & $70-87$ & 49-93 & $56-92$ \\
\hline \multirow[t]{2}{*}{ Social cognition } & Mean (SD) & $73.88(11.5)$ & $72.7(11.0)$ & 74.38 (6.6) \\
\hline & Range & $53-92$ & $47-96$ & $67-85$ \\
\hline \multirow[t]{2}{*}{ Social communication } & Mean (SD) & $76.25(9.2)$ & $72.7(11.7)$ & $80.88(11.1)$ \\
\hline & Range & $66-90$ & $50-96$ & $67-96$ \\
\hline \multirow[t]{2}{*}{ Social motivation } & Mean (SD) & $58.00(13.6)$ & $64.52(11.8){ }^{\#}$ & $69.5(13.6)^{\#}$ \\
\hline & Range & $45-89$ & $41-95$ & 58-95 \\
\hline
\end{tabular}


Table 3 Emotional and behavioural characteristics (Continued)

\begin{tabular}{lllll}
\hline Outcome measure & & Groups & \\
\cline { 3 - 5 } & & STXBP1 full dataset $(n=8)$ & ID all $(n=33)$ & ID low ability $(n=8)$ \\
\hline RRB & Mean (SD) & $79.00(11.9)$ & $78.36(15.2)$ & $87.38(14.7)$ \\
& Range & $62-92$ & $48-108$ & $72-108$ \\
SRS total score & Mean (SD) & $77.75(9.3)$ & $75.12(12.0)$ & $81.75(10.9)$ \\
& Range & $66-94$ & $49-98$ & $70-98$ \\
\hline
\end{tabular}

${ }^{\#}$ Comparison to STXBP1 full dataset group, Mann-Whitney $U$ test, $P<0.05$

including specific phobia, but these symptoms varied across the group. We observed an additional contrast in some individuals between low social anxiety and high non-social anxiety. In view of moderate to severe ID, it is important to consider whether behavioural characteristics within this group differ from developmental expectations-we found that receptive language ability and socialisation skills (but not hyperactivity) discriminated the STXBP1 and ID comparison groups, suggesting a specific contribution of aetiology to these features.

We were struck by a consistent profile of social behaviour within the STXBP1 group. Participants showed great enjoyment of social interactions with family members and demonstrated reciprocal behaviours such as sharing and turn-taking. Researcher experience was that, from the outset of the testing sessions, participants wanted to engage with the researchers (a novel stranger within the participants' home) particularly in social activities such as drawing, dancing, singing, and playing games. Even participants as young as 2 years held eye contact and were very sociable. On the other hand, parental questionnaire ratings indicated limited understanding of social expectations and difficulties integrating into normal social settings. The observation of significantly stronger social motivation remained true, whether comparing to the whole ID sample or low-ability ID sample, indicating that this feature is not simply a correlate of ID severity. Although several individuals scored above cut-off on the SRS for a likely autism spectrum diagnosis, this profile of social behaviours would not be typical

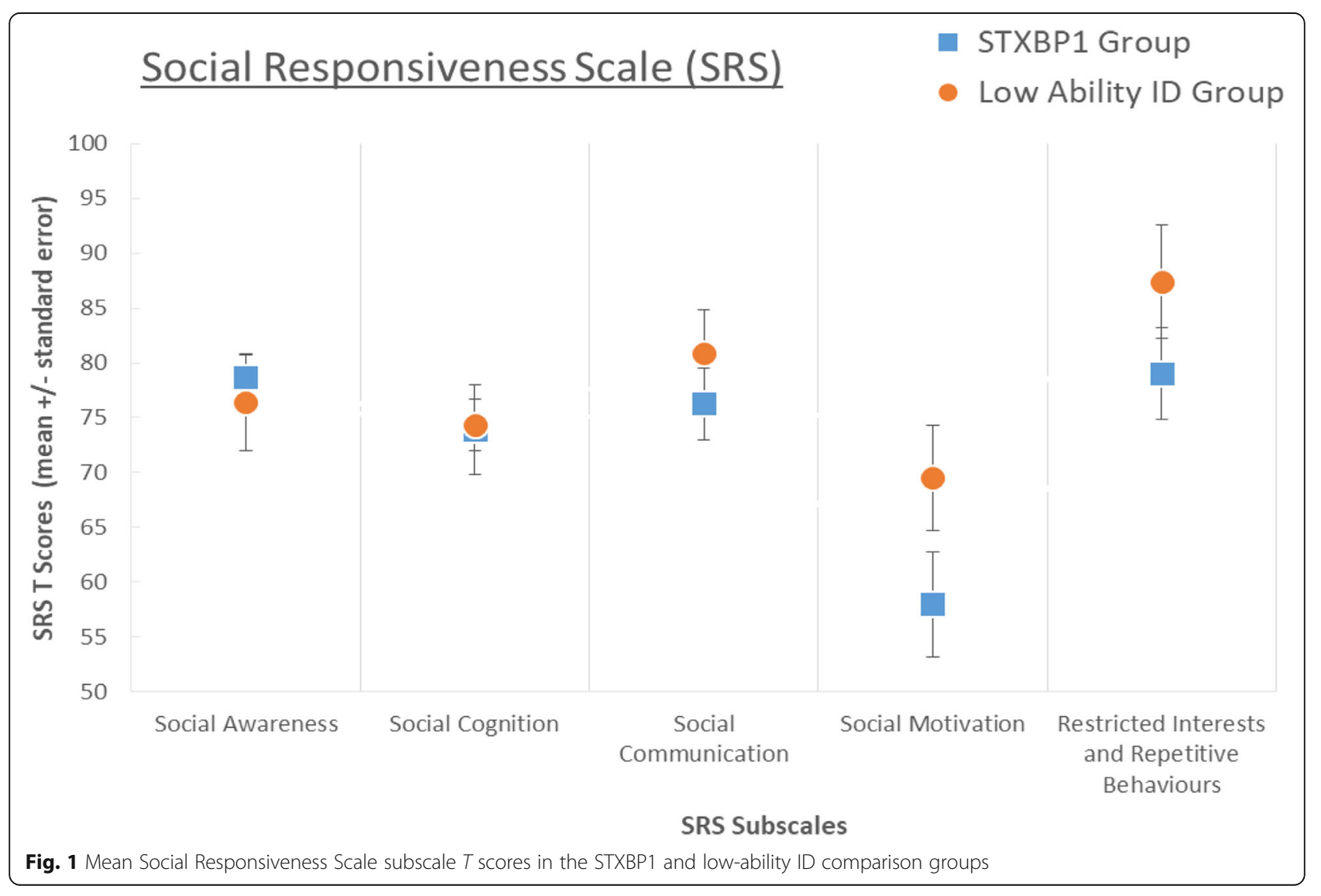


for an individual with autism. These findings support other research into the diverse social developmental trajectories contributing to autism-like impairments in individuals with genomic disorders [31-33].

Epilepsy prevalence within the $S T X B P 1$ group was high, as previously reported, but the type, severity, age of onset, treatment sensitivity, and remission of seizures varied across participants. A relatively high prevalence of focal seizures was reported, in keeping with previous reports [7]. Further studies are needed to examine the contribution of subclinical seizure activity to developmental progress for individuals with STXBP1 variants, including interactions between low-level sensory processing, cognitive biases, and the social environment to mediate anxieties or prosocial behaviour. In addition, comparison to other groups with similar epilepsy prevalence and seizure characteristics could tease apart aetiology-specific behavioural features from correlates of, for example, focal seizures.

A further goal of this research is to stimulate future investigation of the mechanisms linking presynaptic dysfunction to cognitive and social development, which may ultimately lead to aetiology-specific therapies. Toward this goal, we compared our observations of the STXBP1 group to published behavioural phenotyping studies of mice with STXBP1 mutations, to consider consistency across species. The behavioural phenotypes exhibited across different mice models include elevated anxiety, hyperactivity, and impairments in acquiring and maintaining spatial memory and reversal of previously learned strategies $[25,32,34]$. Importantly, not every aspect of learning and behaviour is impaired: STXBP1 haploinsufficient mice demonstrate normal sociability, preference for social novelty, and normal profiles of attentional control without impulsivity [25]. In one study, social behaviours in STXBP1 mouse models were examined by introducing a novel mouse into the chamber [27]. Regardless of specific STXBP1 variant, heterozygous mice spent more time around the novel mouse and displayed normal sociability and preference for social novelty. These findings appear, at least on the surface, to be convergent with our observations of young people with STXBP1 variants, and future research could explore whether similar neurodevelopmental mechanisms underpin social interactions across species.

In considering potential mechanisms underlying this and other aspects of the STXBP1 phenotype, we have been struck by the phenotypic similarity with Angelman syndrome (AS). AS is characterised by developmental delay, seizure susceptibility including focal seizures, movement disorders, language deficits, impulsivity, short attention span, and specific phobias [33, 35-38]. The behavioural hallmarks of AS are hyperactivity and hypersociability [34]. Just as in STXBP1, individuals with AS display increased social motivation, prolonged social interest, and also excessive smiling and laughing [39]. Socialisation is believed to be underpinned by a network of brain regions including the amygdala, ventral striatum, orbital, and ventromedial regions of the prefrontal cortex. However, different regions and networks may have a greater role in specific aspects of sociability [40]. It has been hypothesised that reciprocal changes within striatal circuits give rise to the atypical social novelty profile associated with AS, consistent with experimental evidence for altered striatal dopamine balance in a mouse model [41, 42]. Examining the striatum and its dependent functional neural systems, in the context of the wider 'social' brain network, may provide a starting point for understanding the neural mechanisms driving the atypical social profile in STXBP1.

There are several important limitations to this study. The sample size is small and encompasses a wide age range. We aimed to ascertain participants as broadly as possible, from multiple regions of the UK and multiple medical specialties. In comparison to previous studies, the STXBP1 participant group had a higher proportion of individuals ascertained via clinical genetics rather than paediatric neurology, which may provide a more comprehensive picture of the phenotypic spectrum or may under-represent individuals with early-onset epileptic encephalopathy. The data were collected via parent report, and it was not feasible to corroborate or elaborate via review of medical records. It was also not feasible for researchers collecting data to be blind to genetic diagnoses; however, our use of standardised parent-report questionnaire measures should mitigate potential bias of diagnosis-informed clinician ratings. Non-parametric statistical analyses are included to enhance our descriptive observations and provide an indication of which measures may discriminate between groups. Given the small sample size and large number of measures, adjusting the $p$ value threshold for statistical significance to account for multiple comparisons is not practical, and results should be interpreted with due caution. The robustness of our findings will, we hope, be tested in future independent studies with pre-registered hypotheses and predictions building on our exploratory results. In view of small sample size, within-group analysis of behavioural variation was not justified (for example genotype-phenotype correlations, associations with epilepsy severity or age of onset), and these analyses would improve the prognostic utility of our results. A further limitation of the current study is that we have not directly assessed cognitive abilities in young people with STXBP1 variants and are reliant on parent report of adaptive function and behavioural characteristics. To determine whether specific learning deficits characterise the disorder and contribute to behavioural characteristics, it is necessary to accurately measure relevant aspects of cognition in individuals with moderate to severe ID. However, assessment of cognitive function in individuals with AS or STXBP1 is challenging due to low levels of 
understanding and hyperactivity. To achieve this, cognitive tasks must first be developed that assess the capacity for learning different skills (rather than simply documenting deficits), are intuitive, and are developmentally appropriate. In addition to behavioural methods, application of non-invasive physiological approaches such as wireless EEG in semi-naturalistic settings may be feasible and informative. These methods should be developed now and applied in future once a larger number of individuals have been diagnosed via genome-wide testing and can be followed from early childhood through to adult life.

\section{Conclusion}

This research presents the post-diagnostic evaluation of children and adolescents with STXBP1-associated neurodevelopmental disorder. Individuals with $S T X B P 1$ variants have a wide range of complex adaptive, social and behavioural characteristics. Via comparison to individuals with other ID-related genetic diagnoses, we have highlighted a more limited set of characteristics which may discriminate individuals with STXBP1 from individuals with other monogenic causes of neurodevelopmental disorder. Delineating this spectrum should assist in recognising this disorder and supporting families after diagnosis. Moreover, convergence between $S T X B P 1$ phenotypes across species and across disorders (AS) provides a new starting point for understanding the molecular and neural mechanisms which underlie prosocial development.

\section{Additional files}

Additional file 1: STXBP1 Participant Information. (DOCX $17 \mathrm{~kb}$ )

Additional file 2: Gene groups and participant numbers recruited to the BINGO study. (DOCX $12 \mathrm{~kb}$ )

\section{Abbreviations}

ADHD: Attention deficit hyperactivity disorder; AED: Anti-epileptic drug; DBC: Developmental Behavioural Checklist; ID: Intellectual disability; MABC2: The Movement Assessment Battery for Children 2; SCBU: Special care baby unit; SES: Socioeconomic status; STXBP1: Syntaxin-binding protein 1

\section{Acknowledgements}

We sincerely thank the study participants, their families, and carers for their contributions to this study. We are grateful to the Deciphering Developmental Disorders (DDD) study, the IMAGINE ID study, UK regional genetic centres, diagnostic laboratories, and referring clinicians for enabling us to publicise this study to the families who have taken part.

\section{Authors' contributions}

SOB and ENC acquired, analysed, and interpreted the data. SOB drafted the paper and all other authors substantially revised it. DEA, GS, and KB conceived and designed the study. The DDD study made a significant contribution to the genetic diagnosis of both study groups and facilitated recruitment to the study. All authors read and approved the final manuscript.

\section{Funding}

This study was funded by the Newlife Charity for Disabled Children and supported by the National Institute for Health Research and Medical Research Council UK. The DDD study presents independent research commissioned by the Health Innovation Challenge Fund [grant number
HICF-1009-003], a parallel funding partnership between Wellcome and the Department of Health, and the Wellcome Sanger Institute [grant number WT098051]. The views expressed in this publication are those of the author(s) and not necessarily those of Wellcome or the Department of Health. The research team acknowledges the support of the National Institute for Health Research, through the Comprehensive Clinical Research Network. This study makes use of DECIPHER (http://decipher.sanger.ac.uk), which is funded by the Wellcome Trust.

\section{Availability of data and materials}

All data analysed during this study are included in this published article (and its supplementary information files).

\section{Ethics approval and consent to participate}

This study was approved by the Cambridge Central Research Ethics Committee ('Phenotypes in Intellectual Disability', reference: 11/EE/0330). Parent or Carer gave written informed consent on behalf of participants under the age of 16 . For participants aged 16 and over, lacking capacity to consent, a consultee was appointed. Prior to testing, research assistants ensured the project had been discussed with participants. The DDD study has UK Research Ethics Committee approval (10/H0305/83, granted by the Cambridge South REC, and GEN/284/12 granted by the Republic of Ireland $\mathrm{REC})$.

\section{Consent for publication}

Obtained within study participation consent.

\section{Competing interests}

The authors declare that they have no competing interests.

\section{Author details}

${ }^{1}$ MRC Cognition and Brain Sciences Unit, University of Cambridge, 15 Chaucer Road, Cambridge CB2 7EF, UK. ²Department of Experimental Psychology, University of Oxford, Anna Watts Building, Radcliffe Observatory Quarter, Woodstock Road, Oxford OX2 6GG, UK. ${ }^{3}$ Wellcome Sanger Institute, Wellcome Genome Campus, Hinxton, Cambridgeshire CB10 1SA, UK. ${ }^{4}$ Department of Medical Genetics, University of Cambridge, Cambridge Biomedical Campus, Hills Road, Cambridge CB2 OXY, UK.

Received: 5 April 2019 Accepted: 22 July 2019

Published online: 06 August 2019

\section{References}

1. Gilissen C, Hehir-Kwa JY, Thung DT, Van De Vorst M, Van Bon BWM, Willemsen $\mathrm{MH}$, et al. Genome sequencing identifies major causes of severe intellectual disability. Nature. 2014;511(7509):344-7 Nature Publishing Group.

2. Rizo J. Mechanism of neurotransmitter release coming into focus. Protein Sci. 2018;27(8):1364-91.

3. Saitsu H, Kato M, Mizuguchi T, Hamada K, Osaka H, Tohyama J, et al. De novo mutations in the gene encoding STXBP1 (MUNC18-1) cause early infantile epileptic encephalopathy. Nat Genet. 2008;40(6):782-8.

4. Carvill GL, Weckhuysen S, McMahon JM, Hartmann C, Møller RS, Hjalgrim H, et al. GABRA1 and STXBP1: novel genetic causes of Dravet syndrome. Neurology. 2014;82(14):1245-53.

5. Deprez L, Weckhuysen S, Holmgren P, Suls A, Van Dyck T, Goossens D, et al. Clinical spectrum of early-onset epileptic encephalopathies associated with STXBP1 mutations. Neurology. 2010;75(13):1159-65.

6. Di Meglio C, Lesca G, Villeneuve N, Lacoste C, Abidi A, Cacciagli P, et al. Epileptic patients with de novo STXBP1 mutations: key clinical features based on 24 cases. Epilepsia. 2015;56(12):1931-40.

7. Stamberger H, Nikanorova M, Accorsi P, Angriman M, Benkel-herrenbrueck I, Capovilla G, et al. STXBP1 encephalopathy: a neurodevelopmental disorder including epilepsy. Neurology. 2016;86:954-962.

8. Allen AS, Berkovic SF, Cossette P, Delanty N, Dlugos D, Eichler EE, et al. De novo mutations in epileptic encephalopathies. Nature. 2013;501(7466):21721 Nature Publishing Group.

9. Chan A, Brunga L, Lamoureux S, Faheem M, Drake J, Shlien A, et al. Germline and somatic mutations in STXBP1 with diverse neurodevelopmental phenotypes. Neurol Genet. 2017;3(6):e199.

10. Barcia G, Chemaly N, Gobin S, Milh M, Van Bogaert P, Barnerias C, et al. Early epileptic encephalopathies associated with STXBP1 mutations: could we 
better delineate the phenotype? Eur J Med Genet. 2014;57(1):15-20 Elsevier Masson SAS.

11. Gburek-Augustat J, Beck-Woedl S, Tzschach A, Bauer P, Schoening M, Riess A. Epilepsy is not a mandatory feature of STXBP1 associated ataxia-tremorretardation syndrome. Eur J Paediatr Neurol. 2016;20(4):661-5 Elsevier Ltd.

12. Rauch A, Wieczorek D, Graf E, Wieland T, Endele S, Schwarzmayr T, et al. Range of genetic mutations associated with severe non-syndromic sporadic intellectual disability: an exome sequencing study. Lancet. 2012;380(9854):1674-82.

13. Suri M, Evers JMG, Laskowski RA, O'Brien S, Baker K, Clayton-Smith J, et al. Protein structure and phenotypic analysis of pathogenic and population missense variants in STXBP1. Mol Genet Genomic Med. 2017;5(5):495-507.

14. Yuge K, Iwama K, Yonee C, Matsufuji M, Sano N, Saikusa T, et al. A novel STXBP1 mutation causes typical Rett syndrome in a Japanese girl. Brain Dev. 2018;40(6):493-7 The Japanese Society of Child Neurology.

15. McRae JF, Clayton S, Fitzgerald TW, et al. Prevalence and architecture of de novo mutations in developmental disorders. Nature. 2017;542(7642):433-8.

16. Wright CF, Fitzgerald TW, Jones WD, Clayton S, McRae JF, Van Kogelenberg M, et al. Genetic diagnosis of developmental disorders in the DDD study: a scalable analysis of genome-wide research data. Lancet. 2015;385(9975):1305-14.

17. Hamdan FF, Gauthier J, Dobrzeniecka S, Lortie A, Mottron L, Vanasse M, et al. Intellectual disability without epilepsy associated with STXBP1 disruption. Eur J Hum Genet. 2011;19(5):607-9.

18. Milh M, Villeneuve N, Chouchane M, Kaminska A, Laroche C, Barthez MA, et al. Epileptic and nonepileptic features in patients with early onset epileptic encephalopathy and STXBP1 mutations. Epilepsia. 2011;52(10):1828-34.

19. Mastrangelo M, Peron A, Spaccini L, Novara F, Scelsa B, Introvini P, et al. Neonatal suppression-burst without epileptic seizures: expanding the electroclinical phenotype of STXBP1-related, early-onset encephalopathy. Epileptic Disord. 2013;15(1):55-61.

20. Yamamoto T, Shimojima K, Yano T, Ueda Y, Takayama R, lkeda H, et al. Lossof-function mutations of STXBP1 in patients with epileptic encephalopathy. Brain Dev. 2016;38(3):280-4 The Japanese Society of Child Neurology.

21. Patzke C, Han Y, Covy J, Yi F, Maxeiner S, Wernig M, et al. Analysis of conditional heterozygous STXBP1 mutations in human neurons. J Clin Invest. 2015;125(9):3560-71.

22. Verhage M. Synaptic assembly of the brain in the absence of neurotransmitter secretion. Science. 2000;287(5454):864-9.

23. Toonen RFG, Verhage M. Munc18-1 in secretion: lonely Munc joins SNARE team and takes control. Trends Neurosci. 2007;30(11):564-72.

24. Grone BP, Marchese M, Hamling KR, Kumar MG, Krasniak CS, Sicca F, et al. Epilepsy, behavioral abnormalities, and physiological comorbidities in syntaxinbinding protein 1 (STXBP1) mutant zebrafish. PLoS One. 2016;11(3):1-25.

25. Kovačević J, Maroteaux G, Schut D, Loos M, Dubey M, Pitsch J, et al. Protein instability, haploinsufficiency, and cortical hyper-excitability underlie STXBP1 encephalopathy. Brain. 2018;141(5):1350-74.

26. Sparrow CB. The Vineland Adaptive behavior Scales-Second Edition-VBAS II. In: Major psychological assessment instruments, vol. 2. Needham Heights: Allyn \& Bacon; 2005. p. 199-231.

27. Einfeld SL, Tonge BJ. Developmental Behavior Checklist. 2nd Edi ed. Australia: University of New South Wales and Monash University; 2002.

28. Conners CK. Conner's 3rd Edition Technical Manual. 3rd Edit ed. Toronto: MHS Assessments; 2008.

29. Constantino JN, Gruber CP. The Social Responsiveness Scale Manual, Second Edition (SRS-2). 2nd Edi ed. Torrance: WPS; 2012.

30. Henderson SE, Sugden DA, Barnett A. Movement ABC-2 Movement Assessment Battery for Children - Second Edition. 2nd Edi ed. Pearson Education Ltd: Minneapolis; 2013.

31. Richards C, Powis L, Moss J, Stinton C, Nelson L, Oliver C. Prospective study of autism phenomenology and the behavioural phenotype of PhelanMcDermid syndrome: comparison to fragile $X$ syndrome, Down syndrome and idiopathic autism spectrum disorder. J Neurodev Disord. 2017;9(1):1-15.

32. Orock A, Logan S, Deak F. Molecular and Cellular Neuroscience Munc18-1 haploinsufficiency impairs learning and memory by reduced synaptic vesicular release in a model of Ohtahara syndrome. Mol Cell Neurosci 2018; 88:33-42. Elsevier.

33. Mabb AM, Judson MC, Zylka MJ, Philpot BD. Angelman syndrome: insights into genomic imprinting and neurodevelopmental phenotypes. Trends Neurosci. 2011;34(6):293-303 Elsevier Ltd.

34. Hager T, Maroteaux G, Julsing J, Van VR, Stiedl O. Munc18-1 haploinsufficiency results in enhanced anxiety-like behavior as determined by heart rate responses in mice. Behav Brain Res. 2014;260:44-52 Elsevier B.V.
35. Horsler K, Oliver C. The behavioural phenotype of Angelman syndrome. J Intellect Disabil Res. 2006;50(1):33-53.

36. Bakke KA, Howlin P, Retterstøl L, Kanavin IJ, Heiberg A, Nærland T. Effect of epilepsy on autism symptoms in Angelman syndrome. Mol Autism. 2018;9(1):1-8.

37. Pelc K, Cheron G, Dan B. Behavior and neuropsychiatry manifestations in Angelman syndrome. Neuropsychiatr Dis Treat. 2008;4(3):577-84.

38. Huang HS, Burns AJ, Nonneman RJ, Baker LK, Riddick NV, Nikolova VD, et al. Behavioral deficits in an Angelman syndrome model: effects of genetic background and age. Behav Brain Res. 2013;243(1):79-90 Elsevier B.V.

39. Oliver C, Woodcock K, Adams D. The importance of aetiology of intellectual disability. In: Grant G, Ramcharan P, Flynn M, Richardson M, editors. Learning disability: a life cycle approach to valuing people. Milton Keynes: Open University Press/Wiley; 2010. p. 135-46.

40. Chevallier C, Kohls G, Troiani V, Brodkin ES, Schultz RT. The social motivation theory of autism. Trends Cogn Sci. 2012:16(4):231-9.

41. Stoppel DC, Anderson MP. Hypersociability in the Angelman syndrome mouse model. Exp Neurol. 2017;293:137-43 Elsevier Inc.

42. Berrios J, Stamatakis AM, Kantak PA, Mcelligott ZA, Judson MC, Aita M, et al. Loss of UBE3A from TH-expressing neurons suppresses GABA co-release and enhances VTA-NAc optical self-stimulation. Nat Commun. 2016;7:1-8 Nature Publishing Group.

\section{Publisher's Note}

Springer Nature remains neutral with regard to jurisdictional claims in published maps and institutional affiliations.
Ready to submit your research? Choose BMC and benefit from:

- fast, convenient online submission

- thorough peer review by experienced researchers in your field

- rapid publication on acceptance

- support for research data, including large and complex data types

- gold Open Access which fosters wider collaboration and increased citations

- maximum visibility for your research: over $100 \mathrm{M}$ website views per year

At BMC, research is always in progress.

Learn more biomedcentral.com/submissions 